Instituto Internacional de Investigación y Desarrollo Tecnológico Educativo INDTEC, C.A.

DOI: https://doi.org/10.29394/scientific.issn.2542-2987.2017.2.4.3.46-63

OAI-PMH: http://www.indteca.com/ojs/index.php/Revista Scientific/oai

\title{
Actividades Recreativas para Fortalecer el Rendimiento Escolar de los Estudiantes
}

\author{
Autora: Ayari Carmen Gelvis Gonzalez \\ Universidad Nacional Experimental "Rafael María Baralt", UNERMB \\ ayagel69@hotmail.com \\ Zulia, Venezuela
}

\section{Resumen}

La presente investigación tiene como propósito Proponer las Actividades Recreativas para Fortalecer el Rendimiento Escolar de los Estudiantes de la Escuela Básica Bolivariana "El Muro" en el Municipio Valmore Rodríguez del estado Zulia. Para la realización de la investigación se procede a la revisión bibliográfica, así como al reconocimiento del escenario para la descripción contextual y geográfica. El estudio es de tipo descriptiva, utilizando en método del paradigma cualitativo bajo la una investigación acción participativa, basada en el modelo de Elliot (2010). Para la recolección de información se utilizó la técnica de la entrevista registrando la información en un instrumento denominado registro de campo. Se ejecutó planes de acción para lograr la transformación. El análisis se hizo mediante la sistematización obtenida de los planes de acción transformadora teniendo como conclusión que las Actividades Recreativas constituyen una estrategia de gran significación para los estudiantes, a través de las cuales pueden mejorar su Rendimiento Escolar, las instituciones deben propiciar condiciones para facilitar el desarrollo de estas actividades. escolar.

Palabras clave: actividades; recreativas; fortalecer; rendimiento; 


\title{
Recreational Activities to Strengthen the Students' School Performance
}

\begin{abstract}
The following research is aimed at Proposing Recreational Activities to Strengthen the School Performance of the Students from "Escuela Basica Nacional "El Muro" in Valmore Rodriguez municipality, Zulia state. For the execution of this research, the bibliographical review was carried out, as well as the recognition of the scenario for the contextual and geographical description. The study is descriptive, using the qualitative paradigm method under a participatory action investigation, based on Elliot model (2010). To gather information the interview technique was used recording the information in an instrument named field record. Action plans were executed to achieve transformation. The analysis was carried out through the systematization obtained from the transformational action plans having as conclusion that the Recreational Activities constitute a meaningful strategy for students, through which they can improve their school performance; the institutions must promote conditions to facilitate the development of these activities.
\end{abstract}

Keywords: activities; recreational; strengthen; performance; school. 


\section{Introducción}

La Recreación como Actividad siempre ha estado presente en la vida humana, a lo largo de la historia, lo que por sus características hace que el hombre se manifieste a través de ella. En la actualidad es una disciplina que declara ser formativa y enriquecedora dentro del desarrollo social. Se ha hablado mucho acerca del uso y aprovechamiento del tiempo libre. Es por esto que las actividades recreativas que se planifiquen en las escuelas promuevan el papel protagónico del estudiante y desarrollen su independencia y creatividad, por lo que se necesita tener una idea clara de la forma de proceder y las características de las diferentes actividades en función del desarrollo integral de su personalidad. Existen factores de dirección y coordinación en la realización de las actividades que son decisivos entre lo que se encuentran la dirección de la escuela e instalación que brindan para la ejecución de las actividades recreativas en sus múltiples manifestaciones.

En los centros educativos en el horario de clase no se incluyen actividades recreativas aun sabiendo la preferencia de esta por los estudiantes, se debe tener en cuenta que el logro de una recreación sana es fundamental en esta etapa de la vida escolar. Cuando se habla de una recreación siempre se piensa en nuevas alternativas o cambios.

Es de dicha importancia la planificación y organización de las actividades en este tiempo, pues no todos los docentes poseen los conocimientos y habilidades pedagógicas para desarrollarlas y debido a esto los objetivos propuestos se ven afectados.

\subsection{Contexto Real de la Situación}

La investigación se realizó en la Escuela Básica Integral El Muro, ubicada en la Carretera Bachaquero -El Venado con Avenida Nro. 74 del Municipio Valmore Rodríguez del Estado Zulia, su entorno comunitario presenta características especiales considerando que está situado en una vía 
principal, sector residencial rural, el cual alberga a una población numerosa de 395 estudiantes pertenecientes a diferentes estratos sociales, los cuales van desde grupos en pobreza extrema hasta colectividades con una calidad de vida acorde a las necesidades básicas de cualquier ser humano.

Cabe agregar, la particularidad de la institución educativa la cual se encuentra en el renglón de instituciones Bolivarianas con horario de clases (08:00 am hasta las 04:00 pm), diferente al resto de las escuelas, donde se imparte en las mañanas el contenido curricular dispuesto por el Ministerio del Poder Popular para la Educación, en el horario de la tarde se realizan actividades complementarias tales como: talleres de títeres, danzas, reciclajes, educación deportiva, entre otras.

En este tipo de instituciones los estudiantes reciben una alimentación balanceada, la cual contempla desayuno, almuerzo y merienda, se rigen por el Programa de alimentación Escolar del Gobierno Bolivariano, implementado con la finalidad de mejorar la calidad de vida de los y las estudiantes de entornos con menos poder adquisitivo, es decir, sus familias cuentan con bajos recursos económicos, debido a la carencia de fuentes de empleo formales, la mayoría trabajan en el comercio informal, en casa de familias y en parcelas agrarias.

\subsection{Idea General de la Investigación}

La educación venezolana ha generado transformaciones en todo el proceso de aprendizaje del alumno; Para Gagne (2010), plantea la función educativa de la escuela requiere de una comunidad de vida de participación democrática, de búsqueda intelectual, de dialogo y aprendizaje compartido discusión abierta sobre la bondad, una comunidad que rompa las absurdas barreras arteriales entre la escuela y la sociedad.

Al mismo tiempo, manifiesta que el proceso educativo es de carácter cualitativo e integral basada en los valores del ser humano así como en la 
participación de manera activa, solidaria y consciente de todos los responsables del hecho educativo, en los procesos de aprendizaje del estudiante, transformando a cada individuo socialmente en cuanto a los valores e identidad nacional, responsabilidad, amor, justicia y la libertad, que permita esto contribuir al desarrollo de una conciencia ciudadana para la conservación ambiental, la paz, entre los pueblos, la defensa, el mejoramiento y calidad de vida donde elabore sus propios juicios críticos antes los problemas y conflictos sociales y personales.

En tal sentido, hablar de educación ha sido sinónimo, por mucho tiempo de estímulos y oportunidades para un desarrollo desde sus primeros años de vida. A esto se acuña el criterio de Pulido (2013),

En regiones como América Latina, que en conjunto representa un escenario lleno de dificultadas y amenazas innegables para los niños esta preocupación por la "normalidad" del desarrollo lleva a los educadores a enfatizar de un servicio orientado a la prevención de distorsiones y a la compensación del déficit en el desarrollo educativo.

En consecuencia, la educación ha sido objeto de permanente revisión en su diseño curricular, el cual en la década de 1998 hasta 2015 se han presentado dos cambios considerables, el primero lo generó el diagnóstico realizado por Exministro de Educación el Dr. Cárdenas, quien hizo un llamado a un Plan de acción el mismo se desarrolló en el Currículo Básico Nacional en el año (1998), el cual se implantó significativos cambios que permitieron fortalecer el sistema educativo.

El segundo, llamado Diseño Curricular Bolivariano, que en la actualidad se concibe como un proceso histórico social, integral y permanente, sustentado en el preámbulo de la Constitución de la República Bolivariana de Venezuela CRBV (1999), cuyos cambios se puede señalar en términos generales, como la educación dentro de una perspectiva global; precisamente, se concibe la creencia de que un mayor tiempo escolar real apoyado en otros 
cambios educativos viene a ser una condición ineludible para la corrección de algunos de los más graves problemas confrontados por la educación, lo que ha ido adquiriendo un status privilegiado como política pública y privada, no solamente en Venezuela sino que ha tenido redundancia en otros países de América Latina.

Por consiguiente, en el país existe consenso alrededor de algunas proposiciones sobre los nocivos efectos del doble turno. Asimismo, se acepta que la reducción del tiempo escolar ha producido un deterioro apreciable en las capacidades pedagógicas de la escuela, deterioro que opera con relativa independencia de otros factores como la calidad del entrenamiento recibido por los docentes, la calidad de la gestión pedagógica del personal del plantel, la colaboración de las familias y de la comunidad a través de sus organizaciones, ya que coloca límites invariables a la posibilidad de interacciones significativas con los alumnos y por tanto, se ha de considerar las actividades recreativas a fin de alcanzar el desarrollo integral de los estudiantes.

Al respecto, los docentes transfieren a los estudiantes contenidos curriculares individuales, lo que desvía la educación hacia la realización las tareas, centradas principalmente en el simple suministro e información en detrimento a la función formativa de la personalidad; lo que conlleva a una inadecuada enseñanza, la atención pasiva durante el horario de clases, privando al alumno del tiempo necesario para cumplir con las actividades que sugieren alegría, esparcimiento, socialización, es decir las actividades recreativas que pudieran permitirles consultar textos, para pensar, discutir, observar, criticar, crear, experimentar y contemplar.

Entre tanto, la apropiada capacidad de la familia para sustituir exitosamente a los docentes durante el lapso que los estudiantes dedican a las actividades recreativas y la profundización de su desarrollo integral. Deben entonces, ser transformadas en el complemento del proceso aprender- 
aprender, esto fortalecería el desempeño escolar y como consecuencia el rendimiento estudiantil. Lógicamente, los niños, jóvenes, poseen un gran cúmulo de energías que requieren ser orientadas positivamente en beneficio del buen desarrollo de las actividades dentro del salón de clase que en muchas ocasiones estas energías si no son canalizadas correctamente entorpecen la labor del docente.

Dentro de este orden de ideas Pérez (2010: Pág. 23), expresa: "Las actividades extraescolares son útiles en la medida en que favorecen el desarrollo personal del niño y que éste debe vivirlas como una experiencia lúdica, un tiempo dedicado al juego distinto a las clases convencionales". Cuando se da la oportunidad de participar a los estudiantes en actividades extraescolares, se propicia un ambiente más efectivo para desarrollar el proceso educativo puesto que es la oportunidad para aplicar los conocimientos adquiridos y al mismo tiempo fomentar valores de convivencia entre la población estudiantil, lo cual contribuye con el desarrollo integral de los mismos.

Al momento de elegir una actividad educativa para los alumnos alumnas, es fundamental contar con su opinión favorable, es decir, que estas actividades no pueden ser impuestas por terceras personas, al contrario, se establecen en mutuo acuerdo entre el docente, el padre, representante; de manera que se garantice el entusiasmo y motivación. De lo contrario, al poco tiempo se sentirá desmotivado lo que terminará aburriéndole dicha actividad. Tampoco se puede abusar de la implementación de las actividades extraescolares.

Es importante que en las instituciones educativas se promueva la recreación como prácticas que constituyen elementos fundamentales para alcanzar una vida plena, a través de la formación aptitudes, capacidades, habitas y destrezas que permitan el desarrollo armónico e integral de los individuos. Considerando que el proceso educativo busca más que la 
adquisición de conocimientos, la generación de experiencias significativas que hagan que el individuo modifique su conducta, permitiendo aplicar estos en la vida cotidiana y facilitándole su integración en la sociedad, es decir, un desarrollo integral que fortalezca lo cognitivo, social, afectivo y moral.

En el país, la parte de la actividades recreativas es transferida por los docentes al personal especialista, como son el docente de educación física, música, difusión cultural, educación para el trabajo; sin embargo, es bien conocido que las actividades que estos desarrollan están orientadas hacia ciertos grupos en el aula, esto quiere decir que los alumnos que no se sienten motivados hacia dichas actividades, no son en la mayoría de los casos considerados y se convierten en alumnos rezagados, son esporádicamente tomados en cuenta en el desarrollo de las actividades sin notar que esta afecta negativamente el entusiasmo y autoestima de ellos.

Es por ello, que el alumno aspira a ser representante en cada una de las áreas recreativas desarrolladas; con el principal objetivo de sensibilizarse de manera que aprenda nuevas formas de expresión y que contribuya a su desarrollo psicomotriz; es decir, que la población estudiantil siempre se muestra interesada a participar de manera activa en el desarrollo de actividades extraescolares, ya que lo ve como una manera más entretenida de aprender y expresar sus sentimientos, emociones, sensaciones, lo cual le permite desarrollar su propio cuerpo, sus cualidades y capacidades.

En este orden de ideas, pareciera que los niños después de realizar sus actividades académicas permanecieran en las aulas dejando de aprovechar el tiempo libre, debido a que los docentes en su mayoría dejan de planificar este tipo de actividades extraescolares, muchas veces por no contar con los recursos y espacios físicos para llevarlas a cabo, aunado a esto, la existencia de poco personal especialista, ocasiona que no existan suficientes recursos disponibles para cubrir esas horas a toda la población estudiantil. 
Por ello el desarrollo integral de los estudiantes se ve limitado, ya que solo se preocupa por la parte académica la cual es repetitiva y dejan a un lado la parte afectiva, lo que trae como consecuencia bajo rendimiento estudiantil, quizás por la monotonía del proceso educativo, siendo la mejor manera para lograr el desarrollo integral de los estudiantes la planificación y ejecución de actividades recreativas dentro y fuera del aula ya que estas además de dejar un aprendizaje, propician la integración

Según las observaciones realizadas por la autora del presente proyecto de investigación y teniendo la experiencia como docente de aula pareciera que los aspectos antes señalados se están manifestando en la Escuela Básica Integral Bolivariana EL MURO, por cuanto, con frecuencia en las horas libres se evidencia desmotivación y desinterés por parte de los docentes en procura de desarrollar habilidades y destrezas, dejando de lado la oportunidad de convertir estos espacios en significativa experiencias de aprendizaje y socialización.

No, obstante, en reuniones de docentes se ha manifestado que los docentes deben asumir el currículo en su máxima expresión, donde se conjuguen las áreas de aprendizaje mediante la ejecución de proyectos que inviten a los estudiantes al desarrollo de sus capacidades y habilidades con la finalidad de transferir conocimientos en forma divertida que motive a los mismos a participar y por ende a aprender significativamente. Por lo que esta situación se viene reiterando en la institución y sería una oportunidad de indagar cómo incide esta situación en el proceso de educativo y sus alcances para el rendimiento escolar.

\subsection{Propósitos General de la Investigación.}

Proponer las Actividades Recreativas para Fortalecer el Rendimiento Escolar de los Estudiantes de la Escuela Básica Bolivariana "EL MURO". En el Municipio Valmore Rodríguez del estado Zulia, 


\section{FUNDAMENTACIÓN TEÓRICA}

\subsection{Actividad}

La actividad (Lat. activitas, activas = actuar). Mediatiza la vinculación del sujeto con el mundo real. La actividad es generadora del reflejo psíquico el cual, a su vez, mediatiza a la propia actividad. Siguiendo a Merani (1998: 4), podemos entenderla de la siguiente forma:

Se refiere al conjunto de fenómenos de la vida activa, como los instintos, las tendencias, la voluntad, el hábito, entre otros, que constituye una de las tres partes de la psicología clásica, junto con la sensibilidad y la inteligencia.

En este sentido, el practicar alguna actividad siempre está ligado a cierta necesidad que provoca la búsqueda. Durante la realización de la actividad colectiva e individual tiene lugar el reflejo psíquico de la realidad y se forma la conciencia. La actividad humana consciente tendiente hacia una finalidad es la sustancia de la conciencia humana porque es un proceso objetivo tanto como todos los procesos de la naturaleza.

Dentro de este orden de ideas, en este estudian la actividad externa y la actividad interna donde esta última es secundaria porque se forma en proceso de interiorización de la actividad externa formando un plano interior de la conciencia. Este proceso de interiorización Vigostky lo interpretaba como pasaje de la función psíquica superior desde el plano social externo al plano individual interno de su realización. La actividad está estrechamente interrelacionada con los conceptos conciencia y de lo ideal.

\subsection{Actividades Educativas}

Según lo expresado por Coll, C (2012: Pág. 54) "representan un conjunto de acciones planificadas llevadas a cabo por docentes y estudiantes, dentro o fuera del aula, de carácter individual o grupal, que tienen como finalidad alcanzar los objetivos y finalidades de la enseñanza". Al respecto, se puede decir que las actividades educativas se deben programar en función a 
las necesidades individuales de los estudiantes y tienen como objetivo favorecer el desarrollo de sus habilidades físicas, intelectuales, emocionales y sociales estimulando su curiosidad natural. Es de gran importancia al hecho que los estudiantes estén en contacto con la naturaleza por lo que debe planificarse actividades donde se sale a caminar y a jugar en el aire fresco, estas actividades pueden clasificarse de la siguiente manera:

\subsection{Actividad Motriz}

Desde la perspectiva de Reifs, M (2010: Pág. 126) estas constituyen un conjunto de actividades que contribuyen en la estructuración del esquema corporal, siendo su objetivo fundamental pues les permiten a los estudiantes aumentar progresivamente su competencia motriz, pasando de "ser dominado por sus movimientos" a "dominar su motricidad". El alumno estudiante construye su propio esquema corporal en interacción con el espacio, el tiempo, el movimiento propio y el de los otros.

El niño toma como punto de partida el conocimiento que tiene de las diferentes partes de su cuerpo y de los demás, pasando del movimiento global al segmentario y afirmando definitivamente el proceso de lateralización. Empieza a tomar conciencia de su propio cuerpo, pasando de la acción a la representación, tanto de su cuerpo como de su acción motora.

\subsection{Actividad Cultural}

Bugeda, J. (2014: Pág. 23), propugna, "las actividades culturales, son aquellas que cualquier persona puede desarrollar voluntariamente para relajarse, divertirse, entretenerse, formarse, desarrollar su capacidad creadora, disfrutar del arte, museos, cine, teatro; realizar excursiones; elaborar manualidades, artesanías, entre otros. En el ámbito educativo las actividades culturales promueven en los estudiantes los valores y apego por sus raíces, así como le permiten tener una formación especial la cual les sirve a futuro 


\subsection{Actividad Social}

Para Navarro, V (2012: pág. 165), "se refiere a un número indeterminado de personas que interactúan entre ellas para conseguir un objetivo común y diverso. Las actividades sociales constituyen una de las partes determinantes de la estructura social".

En este sentido, la actividad social es considerada como un sistema de dinamización que permite la participación constante de todos los actores socioeducativo para desarrollar las capacidades creativas del individuo donde se busque el crecimiento participativo en los procesos social de una comunidad

\subsection{Recreación}

Desde la perspectiva teórica de Fritzen (2014: pág. 12) "La recreación es una actividad que relaja a los seres humano de la fatiga del trabajo, a veces proveyéndole un cambio, distracción, diversión y restaurándolas para el trabajo." Cabe destacar que hoy día, no es ligera ni pasiva, sino que involucra procesos que ejercitan desde la perspectiva física, mental y emocional; es decir, más en la recreación que en el trabajo. Actualmente hay una población que no trabaja y hay que hacerle un programa también para ellos. Ya que esto enriquece la calidad de vida y es esencial para el bienestar individual y colectivo.

Para Martinelli (2012: pág. 67) la recreación se caracteriza por ser una forma específica de actividad, una actitud o espíritu, se realiza fuera del trabajo, es una expresión de la naturaleza interna del ser humano, un sistema social, una fase de la educación, una salida para la necesidad creativa, un medio para levantar las alas del espíritu. Además, es divertida, refrescante, asimismo, es una respuesta emocional personal, una reacción psicológica, una actitud, un enfoque una manera de vivir. Ofrece una salida para las 
potencias físicas, mentales o creativas. Que es libre, placentera, su propia atracción.

Si bien es cierto, estas actividades de tipo recreativas son, entonces, experiencias personales voluntarias de las cuales se deriva un sentido de bienestar personal, disfrute y placer que eventualmente ayuda al desarrollo y logro de un equilibrio en las dimensiones físicas, mentales, emocionales, espirituales y sociales del individuo.

En este sentido, la teoría de la recreación como una auto-expresión reconoce la naturaleza del hombre, su estructura anatómica, su inclinación psicológica, su capacidad de sentimiento y su deseo de auto-expresión. Acota además del autor que en consideración del hecho de que las formas de actividad a través del cual el hombre alcanza su disfrute son condicionadas por sus posibilidades mecánicas (habituales) de comportamiento, su condición física, y sus actitudes y hábitos.

2.7. Características de la Recreación, según Martinelli (2012: pág. 69) La recreación posee los siguientes atributos o características:

- Ocurre principalmente en el tiempo libre. El tiempo de la recreación ocurre fuera de las horas de trabajo, cuando el individuo se encuentra libre para escoger su actividad deseada.

- Es voluntaria la persona la elige sin compulsión de imaginación. La recreación no es compulsiva, i.e., proviene de una motivación intrínseca. La persona es libre para seleccionar el tipo de actividad que más le interesa y que le provea satisfacción personal.

- La recreación provee goce y placer. Las actividades recreativas se disfrutan a plenitud. La recreación es en esencia una experiencia personal voluntaria a través de la cual se deriva directamente disfrute y satisfacción. El individuo incurre en una actividad recreativa porque 
recibe satisfacción o placer de las mismas o porque percibe valores sociales o personales.

- Ofrece satisfacción inmediata y directa. La única recompensa para el individuo es la satisfacción que proveen las actividades recreativas. El impulso o deseo que conduce a los participantes en las actividades recreativas proviene del disfrute $y$ placer que se obtiene inmediatamente de la propia actividad.

- Se expresa en forma espontánea y original. Es lúdica, ya que incluye expresiones espontáneas e instintivas, la cual ha de ser del agrado de la persona, es decir, que de dicha actividad se obtendrá satisfacción o placer interno y externo. Se deriva placer de la misma (resulta en satisfacción inmediata e inherente al individuo).

- Ofrece oportunidad de auto-expresión y de ella extrae la diversión. De la recreación se reciben respuestas placenteras y gratificantes.

- Le da la oportunidad al individuo de manifestar su creatividad. La recreación ayuda a la renovación del espíritu. Provee un medio positivo para el mejoramiento de las dimensiones físicas, mentales y morales del individuo.

- Provee un cambio de ritmo de la vida cotidiana. Se refresca el individuo permite mantener un equilibrio de sus dimensiones físicas, mentales, emocionales, sociales y espirituales. Contribuye a una vida satisfaciente, disfrutable y abundante.

- La recreación es sana, constructiva y socialmente aceptable. Esto puede ser un área de controversia, puede lo que es sano para una cultura o país, puede ser denigrante en otro lugar del mundo. Esto implica que las normas morales locales dictan las pautas para lo que son actividades recreativas aceptables y constructivas y aquellas que no lo son. La recreación mejora y enriquece la vida personal. 
- Incluye actividades tanto pasivas como activas.

- Puede ser novedosa

\subsubsection{Recreación escolar}

De acuerdo a la opinión de Ruiz (2015) define la recreación escolar todas aquellas actividades agradables, sometidas a reglas muy sencillas realizadas en un ambiente de alegría y diversión. Estas actividades han sido establecidas atendiendo criterios a saber según el grado de participación puede ser:

Activas: se sobre entiende la participación de la persona es activa.

Pasiva: el participante solo cumple funciones de observador y de oyente.

\section{Según el ámbito de aplicación pueden ser:}

Al aire libre actividades realizadas en medios naturales en lugares descubiertos.

Espacios cerrados actividades realizadas en el marco de una estructura física cubiertas.

\section{Metodología}

Para el desarrollo de este artículo. Se eligió la investigación-acción según Elliont (2010), que refiere que la misma se establece con el conocimiento científico y la solución un problema dirigido al docente para que reflexione sobre su práctica educativa, El modelo propuesto de acuerdo al autor está referente a la ejecución de acción transformadora colectivamente con la participación de los docentes, de igual manera esta investigación está relacionada con las técnicas entre ellas tenemos la observación, entrevista, 
que permitió la sistematización e interpretación de los resultados, por medio del instrumento denominado registro de campo

\section{Resultados de las Categorización}

Que los docentes mediante las acciones refirieron que la aplicación de las actividades recreativas permite en proceso educativo favorable y que a su vez, permite evaluar positivamente a los estudiantes. Así mismo, pueden ser motivados por tener mejor rendimiento escolar.

De igual manera reconocer la importancia de las actividades recreativas para involucrar a los padres, representantes y esta a su vez permite que los alumnos escojan sus actividades en su tiempo a fin de focalizar conocimientos, con la satisfacción que le provee las actividades.

Asimismo, consideran que las actividades recreación es una estrategia que contribuye al desarrollo integral del individuo, involucrando iniciativa, liderazgo, toma de decisiones y autoestima.

De acuerdo a los resultados se puede determinar que mantener la activación de la de las actividades recreativa permitirá fortalecer la relación escuela-docente-comunidad, mediante la interacción de estudiantes, familias, consejos comunales, líderes comunitarios, exestudiantes, organizaciones populares, sindicatos, iglesias, voluntariados entre otros, constituidos a fin de apoyar el centro educativo.

Asimismo, Desarrollar en las y los estudiantes el fortalecimiento, la vida académica, mediante una participación dinámica, de todos los miembros y representantes comunitarios solventando los problemas, y generando propuestas de mediación, partiendo de iniciativas, que desarrollen sus habilidades y destrezas en actividades recreativas.

De igual manera se debe impulsar la dotar de espacios físicos adecuados tanto en la escuela como en la comunidad para desarrollar en armonía y al aire libre las actividades recreativas, donde se contribuya al 
desarrollo de sus habilidades, y destrezas, realizando aportes significativos para lograr los recursos necesarios en la activación de las actividades recreativas para lograr sujetos participativos, democráticos, disciplinados, creativos, convirtiéndose en un vínculo entre escuela-comunidad.

\section{Conclusión}

Tomando en cuenta el propósito Proponer las Actividades Recreativas para Fortalecer el Rendimiento Escolar de los Estudiantes de la Escuela Básica Bolivariana "EL MURO". Se puede concluir en lo siguiente:

Que las actividades recreativas mejoran los beneficios aportados por el docente a los niños y niñas inmersos en el sistema educativo en el nivel de educación primaria. Así como favorecer al docente, ya que éste tiene la responsabilidad de brindar a los niños actividades que promuevan la formación de individuos aptos para vivir, haciendo uso adecuado de acciones pedagógicas acordes a cada uno de ellos, a esto se le añade que fortalece el desarrollo motor en los niños del nivel de educación primaria, ofreciendo al docente herramientas que le permitan cumplir con el compromiso de desarrollar ampliamente el conocimiento y por ende lograr el aprendizaje efectivo en los niños a su cargo.

\section{Referencias}

Bugeda. H. (2013). La Recreación Infantil. Editorial Paidos.

Fritzen, P. (2012). Recreación Fundamentos, didáctica y recursos. Editorial Stadium.

Navarro, V. (2002). Aprender en la vida y en la escuela. Ed. Morata. Madrid. Pérez M. (2016). Importancias de la Actividades Extraescolares en la Educación Primaria. Editorial Océano. Bogotá, Colombia. 


\section{Ayari Carmen Gelvis Gonzalez \\ e-mail: ayagel69@hotmail.com}

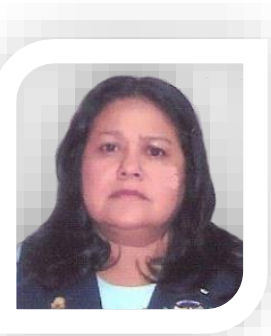

Nacida en Ciudad Ojeda estado Zulia, Venezuela. Cursó estudio de pregrado en la Universidad Nacional Experimental Rafael María Baralt obteniendo título de Lcda. Educación Integral, posteriormente realizo estudios de postgrado en Evaluación por la Universidad Valle del Momboy, estado Trujillo, es Cursante del Doctorado de Educación de la Universidad Nacional Experimental Rafael María Baralt, estado Zulia, Cursante de estudios en Gerencia Educativa de la Universidad Valle de Momboy y Gran Mariscal de Ayacucho, estado Trujillo. Actualmente se desempaña como subdirectora de la Unidad Educativa Nacional Bolivariana Daniel Mavarez, con 13 años al servicio del Ministerio del Poder Popular para la Educación.

El contenido de este manuscrito se difunde bajo una Licencia de Creative Commons ReconocimientoNoComercial-Compartirlgual 4.0 Internacional 